\title{
Intel Software and Programming Tools Ecosystem for HPC
}

Henry A. Gabb

Intel Corporation

High-performance computing (HPC) users are no strangers to code optimization and performance tuning. However, future HPC systems are likely to be even more heterogeneous than they are now. The mix of CPU, GPU, FPGA, and ASIC architectures could be quite diverse. Software will have to be modernized to take advantage of this heterogeneity, and Intel has an extensive ecosystem of programming tools to help. The Intel Fortran and $\mathrm{C} / \mathrm{C}++$ compilers have extensive auto-vectorization capability to deliver maximum performance on Intel processors. Support for the most popular productivity language is provided through the Intel Distribution for Python. Intel also provides a wide range of performance libraries, e.g.: the Intel Math Kernel Library (FFT and numerical linear algebra), the Intel Integrated Performance Primitives (compression/decompression, image, vision, and signal processing), and the Intel Data Analytics Acceleration Library (big data analytics and machine learning). Many Python modules, machine learning frameworks, and third-party libraries already take advantage of the Intel performance libraries. Finally, Intel offers programming tools to support parallel debugging and tuning at the vector, thread, and process level. The key features of each tool will be discussed. 